\title{
DETERMINAÇÃO DE BENZENO, TOLUENO, ETILBENZENO E XILENOS EM GASOLINA COMERCIALIZADA NOS POSTOS DO ESTADO DO PIAUÍ
}

\author{
Flamys Lena do N. Silva, José Ribeiro dos Santos Jr., José Machado Moita Neto e Rosa Lina G. do N. P. da Silva* \\ Departamento de Química, Universidade Federal do Piauí, 64049-550 Teresina - PI, Brasil \\ Danilo Luiz Flumignan e José Eduardo de Oliveira \\ Centro de Monitoramento e Pesquisa da Qualidade de Combustíveis, Biocombustíveis, Petróleo e Derivados, Departamento de \\ Química Orgânica, Instituto de Química, Universidade Estadual Paulista “Júlio de Mesquita Filho", 14800-900 Araraquara - SP, \\ Brasil
}

Recebido em 28/11/07; aceito em 14/7/08; publicado na web em 2/12/08

\begin{abstract}
DETERMINATION OF BENZENE, TOLUENE, ETHYLBENZENE AND XYLENES IN COMMERCIAL GASOLINE FROM PIAUI. STATE. Automotive gasoline consists of a complex mixture of flammable and volatile hydrocarbons derived from crude oil with carbon numbers within the range of 4-12 and boiling points range of $30-225^{\circ} \mathrm{C}$. Its composition varies with the kind of crude oil and the type of refinery process that they undergone. Aromatics hydrocarbons, in particular benzene, toluene, ethylbenzene and isomeric xylenes (BTEX) are the toxic group constituents presents. GC-FID was employed to quantify these hydrocarbons in 50 commercial gasoline samples from Piauí state. Statistical analysis techniques, such as PCA and HCA were used to analyze the data. Moreover, several validation parameters were evaluated.
\end{abstract}

Keywords: BTEX; gasoline; statistical analysis.

\section{INTRODUÇÃO}

A gasolina automotiva consiste na mistura complexa de hidrocarbonetos voláteis e inflamáveis derivados do petróleo, predominantemente com 4 a 12 átomos de carbono e temperatura de ebulição entre 30 a $225^{\circ} \mathrm{C} .{ }^{1,2}$ Sua composição depende da sua utilização, origem e dos processos de refino do petróleo. A gasolina que sai da refinaria apresenta constituição balanceada para atender as especificações de comercialização. $^{3}$

Os hidrocarbonetos aromáticos, em particular benzeno, tolueno, etilbenzeno e os isômeros do xileno (BTEX) constituem um grupo de substâncias presentes na gasolina. ${ }^{4,5}$ Os maiores problemas de contaminação pela gasolina são atribuídos a esses hidrocarbonetos monoaromáticos, que são poderosos depressores do sistema nervoso central. O benzeno é considerado carcinogênico humano por diversas organizações, tais como International Agency of Research of the Cancer (IARC) e a Environmental Protection Agency (EPA). ${ }^{6-8}$

A qualidade dos combustíveis é avaliada pela análise de várias características físicas e químicas, estabelecidas nas Normas Brasileiras (NBR) e Métodos Brasileiros (MB) da Associação Brasileira de Normas Técnicas (ABNT) e de normas da American Society for Testing and Materials (ASTM). A Agência Nacional de Petróleo, Gás natural e Biocombustíveis (ANP) conforme a Portaria $n^{\circ} .309$, de 27/12/2001 estabelece especificações máximas e mínimas de características do combustível, para garantir a qualidade dos derivados do petróleo. O Regulamento técnico da ANP n ${ }^{\circ}$. 05/2001 especifica a gasolina tipo C com $25 \pm 1 \%$ (v/v) de álcool etílico anidro combustível (AEAC) e, no máximo, 1 e $45 \%$ (v/v) de benzeno e compostos aromáticos, respectivamente. ${ }^{9,10}$

A cromatografia gasosa é uma das técnicas mais empregada para quantificar misturas de compostos orgânicos, inclusive benzeno. ${ }^{11-14}$ As normas da ASTM geralmente indicam sistemas complexos de

*e-mail: rosalina@ufpi.edu.br multi-colunas e multi-válvulas, cromatógrafos gasosos acoplados com detector por condutividade térmica (DCT), detector por ionização em chama (DIC), detector por espectrometria de massas (EM) ou a combinação desses. Novos métodos cromatográficos, como por exemplo, CG x CG têm se destacado na análise de gasolina. ${ }^{15,16}$ Além disso, a ASTM desenvolveu uma série de métodos para análise de BTEX em gasolina como mostra a Tabela 1S (Material Suplementar). ${ }^{17-21}$

A cromatografia gasosa quantitativa compara as áreas ou alturas dos picos cromatográficos do analito de interesse com padrões analíticos correspondentes. ${ }^{22,23}$ Neste trabalho empregaram-se na análise quantitativa as áreas dos picos cromatográficos do analito de interesse.

Usou-se o método do padrão externo, que consiste na preparação de várias soluções da substância de interesse a ser quantificada em várias concentrações, em seguida obtiveram-se os picos cromatográficos de cada padrão. ${ }^{24-26} \mathrm{O}$ gráfico de calibração relaciona as áreas dos picos cromatográficos com as respectivas concentrações, podendo ser empregado na determinação dos compostos aromáticos (BTEX). Além disso, o detector DIC exibe sensibilidade alta $\left(\sim 10^{-13}\right.$ $\left.\mathrm{g} \mathrm{s}^{-1}\right)$, larga faixa linear de resposta $\left(\sim 10^{-7}\right)$ e baixo ruído, por isso é excelente para análise de compostos orgânicos. ${ }^{27,28}$

Em nosso trabalho, empregamos a cromatografia gasosa (CG) com detector DIC para determinação de benzeno, tolueno, etilbenzeno e os isômeros do xileno (orto, meta e para) na gasolina comercializada nos postos do estado do Piauí. Os dados obtidos foram tratados por técnicas de análise multivariada permitindo traçar um perfil dessas amostras.

\section{Tratamento multivariado de dados}

A análise de agrupamento hierárquico (HCA) pode ser empregada para agrupar amostras semelhantes entre si, de acordo com as variáveis consideradas relevantes. ${ }^{29} \mathrm{O}$ método HCA categoriza amostras pelo conjunto de valores das variáveis, permitindo o agrupamento de amostras 
similares. Os dendrogramas consistem na representação bidimensional da similaridade entre pares de amostras ou grupos de amostras com escala de zero (amostra similares) a 25 (amostras sem similaridade). ${ }^{29}$

A análise de componente principal (PCA) consiste essencialmente em reescrever as coordenadas das amostras em outro sistema de eixo. Em outras palavras, as n-variáveis originais geram, através de suas combinações lineares, $n$-componentes principais, cuja principal característica, além da ortogonalidade, é que são obtidos em ordem decrescente de máxima variância, ou seja, a componente principal 1 detém mais informações estatística que a componente principal 2, que por sua vez tem mais informação estatística que a componente principal 3 e assim por diante. Portanto, a PCA permite a redução da dimensionalidade dos pontos representativos das amostras já que as últimas componentes principais têm pouca contribuição estatística para a análise dos dados. ${ }^{29} \mathrm{~A}$ construção de gráficos bidimensionais com maior número de informações estatísticas, pode ser alcançada através do gráfico das duas primeiras componentes principais.

\section{PARTE EXPERIMENTAL}

\section{Reagentes}

Os reagentes, todos de grau analítico, benzeno, tolueno, o-, m- e p-xileno foram adquiridos da Merck; o etilbenzeno 99,5\% (o padrão grau cromatográfico) foi obtido da Fluka e o solvente Hexano p.a. para determinação de compostos orgânicos foi adquirido da Mallinckrodt Chemicals.

\section{Equipamentos}

Foi usado um cromatógrafo gasoso Shimadzu modelo 17A equipado com um injetor automático split/splitless modelo AOC-20i, um detector por ionização em chama (DIC) e interfaceado com o software GCsolution v.2.1. como estação de trabalho.

\section{Coluna e condições cromatográficas}

As especificações da coluna, incluindo os fluxos dos gases, as rampas de temperatura da coluna e tipo de injetor estão listadas na Tabela 1 e foram estabelecidas por Flumignan et al.. ${ }^{30,31}$

\section{Preparo das soluções padrões}

As soluções padrões de benzeno, tolueno, etilbenzeno, o-xileno e p-xileno foram preparadas em hexano, na faixa de concentração esperada para cada analito na gasolina e igual a $0,1-3,0 \%(\mathrm{v} / \mathrm{v})$, enquanto o padrão de m-xileno foi diluído na faixa $0,1-2,0 \%(\mathrm{v} / \mathrm{v})$. Todas as
Tabela 1. Condições cromatográficas de análise de gasolina por CG-DIC ${ }^{30,31}$

Condições de análise cromatográfica (CG-DIC)

$50 \mathrm{~m}$ x 0,15 mm x 0,42 $\mu \mathrm{m}$, Shimadzu

Coluna CBP1-PONA Capilar de Sílica Fundida, fase estacionária de dimetilpolissiloxano

Gás de arraste

$\mathrm{He}, 380 \mathrm{kPa}$

Injeção

Split 1:250 (depois de $3 \mathrm{~min}$, razão $1: 30), 0.4 \mu \mathrm{L}, 250^{\circ} \mathrm{C}$

Programação da

Temperatura da Coluna

$30{ }^{\circ} \mathrm{C}, 0,1{ }^{\circ} \mathrm{C}$ min $^{-1}$ até $32{ }^{\circ} \mathrm{C}$,

$2,4^{\circ} \mathrm{C} \min ^{-1}$ até $110^{\circ} \mathrm{C}$, e $6,5^{\circ} \mathrm{C} \min ^{-1}$ até $215^{\circ} \mathrm{C}(10 \mathrm{~min})$.

Hélio, $20 \mathrm{~mL} \mathrm{~min}^{-1}(60 \mathrm{KPa})$

Fluxo de Gases (KPa)

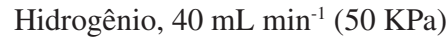
Ar Sintético, $400 \mathrm{~mL} \mathrm{~min}^{-1}$ (40 KPa)

Detector

DIC, $250{ }^{\circ} \mathrm{C}$

amostras foram preparadas em capela e conservadas sob refrigeração e, além disso, os materiais volumétricos utilizados (micropipetas, pipetas e balões volumétricos) apresentavam calibração RBC. Para limpeza de vidraria, empregou-se álcool etílico comercial e acetona p.a. como solvente.

\section{Coleta e preparo das amostras}

Um conjunto de 44 amostras de gasolina comercial tipo C, que consiste na gasolina A adicionada de $25 \pm 1 \%$ de álcool etílico anidro combustível, foram fornecidas pelo Laboratório de Combustíveis (LAPETRO). Dentre as amostras de gasolina comercial, 22 são da região norte do estado do Piauí e 22 da capital Teresina. Em acréscimo, 6 amostras de gasolina das refinarias brasileiras - Manguinhos (R1), Recap (R2), Reduc (R3), Replan (R4), Revap (R5) e Rlan (R6) - foram obtidas junto ao Centro de Monitoramento e Pesquisa da Qualidade de Combustíveis (CEMPEQC). Todas as amostras das refinarias também eram do tipo $\mathrm{C}$ conforme o Regulamento ANP $n^{\circ}$. 05/2001.

\section{Calibração}

Um volume fixo de $0,4 \mu \mathrm{L}$ de cada solução padrão de BTEX e gasolina foram injetados separadamente no cromatógrafo. Todas as análises foram realizadas em triplicata e sob mesmas condições de temperatura. Os parâmetros das curvas são mostrados na Tabela 2.

Tabela 2. Parâmetros da curva para cada composto aromático

\begin{tabular}{|c|c|c|c|c|c|c|c|c|}
\hline Substância & $r^{2}$ & SD & B & A & $\mathrm{S}_{\mathrm{b}}$ & As & $\mathrm{LM} \mathrm{S}_{\mathrm{b}}$ & $\mathrm{LM} \mathrm{S}_{\mathrm{a}}$ \\
\hline Benzeno & 0,9983 & 1480,5871 & 25737,0214 & $-180,8785$ & 447,4476 & 789,7652 & 984,8320 & 1738,2730 \\
\hline Tolueno & 0,9997 & 599,5805 & 25392,5457 & 268,6689 & 181,1990 & 319,8243 & 398,8190 & 703,9330 \\
\hline Etilbenzeno & 0,9997 & 573,6567 & 24662,1222 & $-196,3611$ & 173,3645 & 305,9962 & 381,5750 & 673,4980 \\
\hline o-Xileno & 0,9995 & 892,8743 & 26907,5312 & $-77,5624$ & 269,8352 & 476,2713 & 593,9070 & 1048,2730 \\
\hline m-Xileno & 0,9999 & 147,7756 & 24165,3318 & $-47,9466$ & 79,3428 & 94,1401 & 174,6335 & 207,2023 \\
\hline p-Xileno & 0,9998 & 460,9765 & 24118,0722 & $-369,7088$ & 139,3115 & 245,8911 & 306,6246 & 541,2063 \\
\hline
\end{tabular}

$\mathrm{r}^{2}$ = Coeficiente de Correlação; SD = Desvio Padrão da Curva; B = Inclinação; A = Interseção; $\mathrm{S}_{\mathrm{b}}=$ Desvio padrão da inclinação da reta; $\mathrm{S}$ = Desvio Padrão da Interseção da reta; LM S $_{\mathrm{b}}=$ limite de confiança da inclinação da reta; LM S $\mathrm{a}=$ Limite de confiança para a interseção da reta 


\section{RESULTADOS E DISCUSSÃO}

\section{Validação do método}

Os valores de limite de detecção (LD) e limite de quantificação (LQ), apresentados na Tabela 3, foram calculados pelo desvio médio dos padrões das curvas de calibração. ${ }^{32,33}$ A precisão (repetitividade) e a linearidade foram determinadas por meio da injeção em triplicata de padrões analíticos nas faixas de concentração $0,1-3,0 \% \mathrm{v} / \mathrm{v}$. Os coeficientes de correlação linear variaram de 0,998 a 0,999 como mostra a Tabela 2 .

Todas as amostras apresentaram concentrações superiores ao limite de quantificação calculado. A dispersão de resultados entre as amostras foi medida pelo desvio padrão (s) e o coeficiente de variação $(\mathrm{CV} \%) .{ }^{14}$ Os resultados analíticos estão indicados na Tabela 4.

Os maiores coeficientes de variação entre as amostras foram observados para tolueno e etilbenzeno. A Tabela 4 refere-se ao conjunto das 50 amostras.

Tabela 3. Resultados analíticos de validação

\begin{tabular}{lccc}
\hline Substância & LD $(\% \mathrm{v} / \mathrm{v})$ & LQ $(\% \mathrm{v} / \mathrm{v})$ & Precisão \\
\hline Benzeno & 0,028 & 0,094 & 0,0093 \\
Tolueno & 0,035 & 0,118 & 0,0117 \\
Etilbenzeno & 0,025 & 0,082 & 0,0082 \\
o-Xileno & 0,026 & 0,086 & 0,0082 \\
m-Xileno & 0,013 & 0,043 & 0,0043 \\
-Xileno & 0,049 & 0,163 & 0,0163 \\
\hline
\end{tabular}

$\mathrm{LD}=$ limite de detecção; $\mathrm{LQ}=$ limite de quantificação

\section{Perfil cromatográfico}

A Figura 1S (Material Suplementar) mostra um perfil cromatográfico gasoso típico de uma amostra comercial de gasolina brasileira. Nas condições desenvolvidas por CG-DIC, foi obtida uma boa resolução para os BTEX, benzeno $\left(t_{r}=17,22 \mathrm{~min}\right)$, tolueno $\left(t_{r}=31,60\right.$ $\min )$, etilbenzeno $\left(t_{r}=42,53 \mathrm{~min}\right)$ e xilenos $\left(\mathrm{m}\right.$-xileno, $\mathrm{t}_{\mathrm{r}}=43,4 \mathrm{~min}$; $\mathrm{p}$-xileno, $\mathrm{t}_{\mathrm{r}}=43,5$ min e o-xileno, $\left.\mathrm{t}_{\mathrm{r}}=45,6 \mathrm{~min}\right) .{ }^{32,33}$ Como pode ser observado neste perfil, no benzeno existe uma pequena coeluição com o metilciclopentano (pequeno ombro no pico 1 - Figura $1 \mathrm{~S}$ ), porém este fato não influencia na quantificação real de benzeno em amostras de gasolinas comerciais. Esta confirmação se baseia em resultados satisfatórios obtidos em avaliações realizadas por programas interlaboratoriais de monitoramento de qualidade de combustíveis (PMQC) coordenado pela Agência Nacional do Petróleo, Gás Natural e Biocombustíveis (ANP).

\section{Análise estatística multivariada}

Utilizando o SPSS (Statistical Package for the Social Sciences) e as áreas dos picos dos compostos BTEXs para 44 amostras de gasolina comercial e 6 amostras de gasolina de refinarias, procedeu-se à análise de agrupamento hierárquico por análise de componentes principais.

A Figura 1 mostra o dendograma referente à similaridade das amostras de acordo com as variáveis selecionadas.O primeiro grupo, contém a maioria das amostras e as refinarias R2; R3 e R5. O segundo grupo contém as amostras A6; A9; A11 e A17 e as refinarias R4 e R6. O terceiro grupo, bem diferente dos outros, contém as amostras A19, A20 e a refinaria R1.

A Figura 2 mostra o gráfico da componente principal 1 versus a componente principal 2. O gráfico de escores da componente principal 1 (CP1) versus a componente principal 2 (CP2) explica 90,66\% da variação total dos dados (Tabela 5). A primeira componente principal explica 70,86\% de variância total dos dados e a segunda componente principal explica 19,79\% de variância total dos dados.

A amostra A20 destaca-se na parte positiva da componente principal 1, por apresentar maior concentração de etilbenzeno $(1,36 \% \mathrm{v} / \mathrm{v})$. A amostra A19 destaca-se na parte positiva da componente principal 1 por apresentar maiores teores de o-xileno $(1,5 \% \mathrm{v} / \mathrm{v})$, m-xileno $(3,4 \%$ v/v) e p-xileno (1,2\% v/v). A amostra R1 (Refinaria Maguinhos) apresenta valor maior de tolueno $(3,79 \% \mathrm{v} / \mathrm{v})$ e por isso encontra-se destacada na parte positiva da componente principal 1 .

A amostra R4 (Refinaria Replan) destaca-se das demais amostras na parte positiva da componente principal 2 , devido à maior concentração de benzeno $(0,53 \%$ v/v), seguida pela amostra A8 com $0,48 \%$ v/v de benzeno e as amostras A18 (0,28\% v/v), A1 (0,32\% v/v) e A20 $(0,33 \% \mathrm{v} / \mathrm{v})$ destacam-se na parte negativa da componente principal 2 por apresentarem baixas concentrações de benzeno. A amostra A37 destaca-se na parte negativa da componente principal 1 porque contém os menores teores de etilbenzeno $(0,52 \% \mathrm{v} / \mathrm{v})$, orto-xileno $(0,7 \% \mathrm{v} / \mathrm{v})$, meta-xileno $(1,5 \% \mathrm{v} / \mathrm{v})$ e para-xileno $(0,6 \% \mathrm{v} / \mathrm{v})$.

A análise de componentes principais mostra as amostras A19, A20 e R1 na porção direita da componente principal 1, indicando que essas amostras são ricas em tolueno, etilbenzeno e xilenos, menos em benzeno. Na parte superior da componente principal 2 caracterizam-se as amostras ricas em benzeno. Deste modo, a análise de componentes principais em combinação com a análise de agrupamento hierárquico mostra o seguinte quadro: a maioria das amostras que se assemelham entre si pertence ao grupo I e podem ser provenientes das refinarias $\mathrm{R} 2$; R3 e R5; as amostras com alto teor de benzeno pertencem ao grupo 2 e podem ser provenientes das refinarias R4 e R6; as amostras A19, A20 e R1 são ricas em aromáticos, (menos em benzeno) e pouco se assemelham. Pertence a este grupo a gasolina da refinaria R1.

O estudo comparativo revela que a variabilidade entre os valores de BTEX é maior nas refinarias que nas gasolinas comerciais, indicando que a procedência das amostras comerciais é menos diversificada, como mostra a Tabela 6. O desvio padrão de cada variável (benzeno,

Tabela 4. Valores de medidas estatísticas repetidas para 50 amostras

\begin{tabular}{|c|c|c|c|c|c|}
\hline SUBSTÂNCIA & CON. MÍN. $(\% v / v)$ & CON. MÁX. $(\% v / v)$ & MÉDIA & $D P$ & $C V$ \\
\hline Benzeno & 0,280 & 0,530 & 0,402 & 0,044 & 11,071 \\
\hline Tolueno & 1,590 & 3,790 & 2,035 & 0,377 & 18,499 \\
\hline Etilbenzeno & 0,520 & 1,360 & 0,781 & 0,175 & 22,451 \\
\hline o-Xileno & 0,700 & 1,500 & 0,884 & 0,150 & 17,002 \\
\hline m-Xileno & 1,500 & 3,400 & 1,958 & 0,336 & 17,176 \\
\hline p-Xileno & 0,600 & 1,200 & 0,724 & 0,114 & 15,677 \\
\hline
\end{tabular}




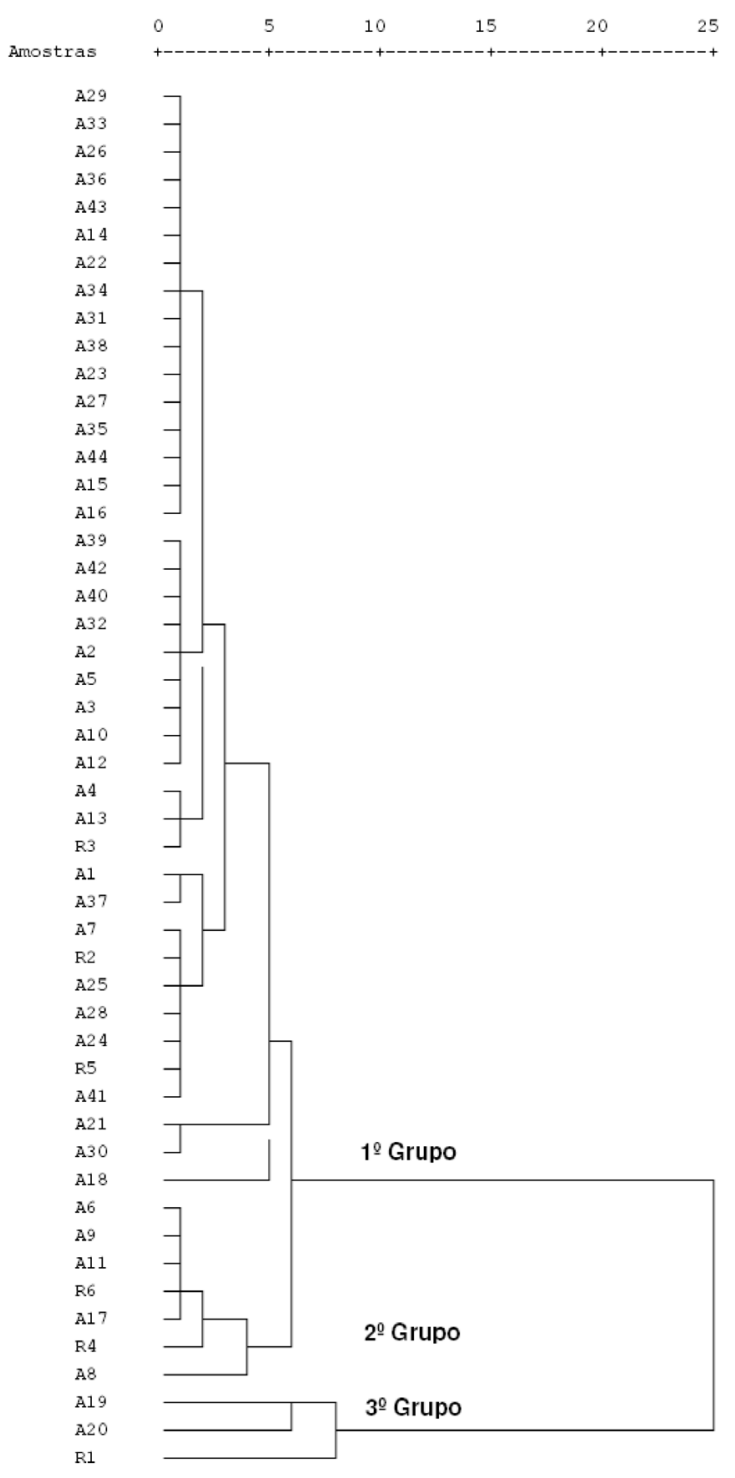

Figura 1. Dendograma obtido através da análise de agrupamento hierárquico de 50 amostras empregando as variáveis benzeno, tolueno, etilbenzeno, oxileno, m-xileno e p-xileno

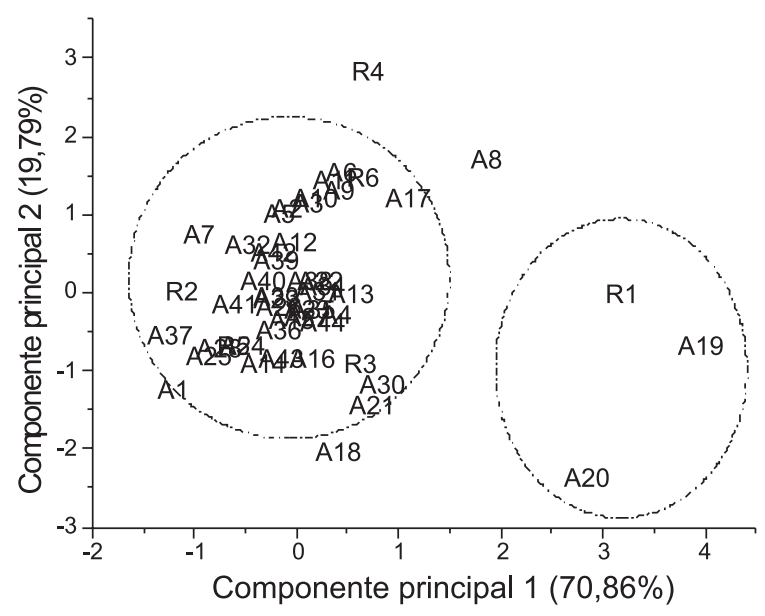

Figura 2. Gráfico dos escores da componente principal 1 versus a componente principal 2 das amostras individuais
Tabela 5. Pesos das variáveis nas componentes principais (CP)

\begin{tabular}{lcc}
\hline Variáveis & \multicolumn{2}{c}{ Componente Principal } \\
& $\mathrm{CP} 1(70,86 \%)$ & $\mathrm{CP} 2(19,79 \%)$ \\
\hline Benzeno & 0,151 & 0,942 \\
Tolueno & 0,841 & 0,358 \\
Etilbenzeno & 0,776 & $-0,402$ \\
o-Xileno & 0,977 & $-0,090$ \\
m-Xileno & 0,987 & $-0,003$ \\
-Xileno & 0,995 & $-0,039$ \\
\hline
\end{tabular}

tolueno, etilbenzeno, orto-xileno, meta-xileno, para-xileno) é menor dentro do grupo que no conjunto total de amostras.

Analisando-se a similaridade entre as refinarias, observa-se que

Tabela 6. Valores das medidas estatísticas para gasolinas comerciais e de refinarias

\begin{tabular}{|c|c|c|c|}
\hline Composto & Parâmetro & $\begin{array}{c}\text { Gasolina } \\
\text { Comercial }\end{array}$ & $\begin{array}{c}\text { Gasolina } \\
\text { de Refinaria }\end{array}$ \\
\hline & Média (\%v/v) & 0,40 & 0,41 \\
\hline & $\mathrm{N}$ & 44 & 6 \\
\hline \multirow[t]{5}{*}{ Benzeno } & DP & 0,04 & 0,07 \\
\hline & Máxima (\%v/v) & 0,48 & 0,53 \\
\hline & Mínima (\%v/v) & 0,28 & 0,35 \\
\hline & Média (\%v/v) & 1,99 & 2,37 \\
\hline & $\mathrm{N}$ & 44 & 6 \\
\hline \multirow[t]{5}{*}{ Tolueno } & DP & 0,28 & 0,74 \\
\hline & Máxima (\%v/v) & 2,96 & 3,79 \\
\hline & Mínima (\%v/v) & 1,59 & 1,77 \\
\hline & Média (\%v/v) & 0,78 & 0,80 \\
\hline & $\mathrm{N}$ & 44 & 6 \\
\hline \multirow[t]{5}{*}{ Etilbenzeno } & DP & 0,17 & 0,23 \\
\hline & Máxima (\%v/v) & 1,36 & 1,24 \\
\hline & Mínima (\%v/v) & 0,52 & 0,57 \\
\hline & Média (\%v/v) & 0,88 & 0,90 \\
\hline & $\mathrm{N}$ & 44 & 6 \\
\hline \multirow[t]{5}{*}{ o-Xileno } & DP & 0,15 & 0,17 \\
\hline & Máxima (\%v/v) & 1,51 & 1,17 \\
\hline & Mínima (\%v/v) & 0,69 & 0,68 \\
\hline & Média (\%v/v) & 1,94 & 2,06 \\
\hline & $\mathrm{N}$ & 44 & 6 \\
\hline \multirow[t]{5}{*}{ m-Xileno } & DP & 0,32 & 0,43 \\
\hline & Máxima (\%v/v) & 3,37 & 2,74 \\
\hline & Mínima (\%v/v) & 1,51 & 1,53 \\
\hline & Média (\%v/v) & 0,72 & 0,77 \\
\hline & $\mathrm{N}$ & 44 & 6 \\
\hline \multirow[t]{3}{*}{ p-Xileno } & DP & 0,11 & 0,16 \\
\hline & Máxima (\%v/v) & 1,18 & 1,04 \\
\hline & Mínima (\%v/v) & 0,57 & 0,58 \\
\hline
\end{tabular}


a refinaria fornecedora do Piauí (R6) apresenta constituição parecida com a REPLAN de São Paulo.

No Brasil, os postos de abastecimento costumam ser classificados quanto ao vínculo á distribuidora. Se existe fidedignidade à distribuidora, o posto tem bandeira, caso contrário é bandeira branca.

Das 44 amostras de gasolinas comerciais analisadas, 12 pertenciam a postos bandeira branca, ou seja, aqueles que não ostentam a marca de qualquer empresa e, portanto, podem adquirir seu combustível de qualquer distribuidora. A maioria é procedente do norte do estado do Piauí. As gasolinas mais divergentes em BTEX pertencem a postos bandeira branca.

\section{CONCLUSÃO}

A gasolina comercial do estado do Piauí conforme a portaria da ANP n ${ }^{\circ} .309$ contém concentrações de BTEX relativamente baixas e inferiores a $1 \%$ de benzeno e $45 \%$ de compostos aromáticos totais. As gasolinas comerciais não são relativamente diferentes das gasolinas de refinarias nos teores de BTEX. Dentre as gasolinas que se destacaram quanto à concentração de BTEX, duas são de refinarias e duas são comerciais de postos bandeira branca.

\section{MATERIAL SUPLEMENTAR}

No material suplementar, disponível gratuitamente em http:// quimicanova.sbq.org.br na forma de arquivo PDF, estão apresentadas a Figura 1S, referente ao perfil cromatográfico típico de uma amostra comercial de gasolina brasileira. e a Tabela $1 \mathrm{~S}$, referente a uma série de métodos oficiais americanos (ASTM) utilizados em análise de combustíveis automotivos.

\section{AGRADECIMENTOS}

À Universidade Federal do Piauí, à Fundação para o Desenvolvimento da UNESP, à Agência Nacional do Petróleo, Gás Natural e Biocombustíveis, à Coordenação de Aperfeiçoamento de Pessoal de Nível Superior, ao Conselho Nacional de Desenvolvimento Científico e Tecnológico e à Fundação de Amparo à Pesquisa do Estado do Piauí, pelo auxílio financeiro e pelas bolsas concedidas aos autores.

\section{REFERÊNCIAS}

1. Wiedemann, L. S. M.; d’Avila, L. A.; Azevedo, D. A.; J. Braz. Chem. Soc. 2005, 16, 139.

2. Flumignan, D. L.; Anaia, G. C.; Ferreira, F. de O.; Tininis, A. G.; de Oliveira, J. E.; Chromatographia 2007, 65, 617.

3. Flumignan, D. L.; Tininis, A. G.; Ferreira, F. de O.; de Oliveira, J. E.; Anal. Chim. Acta 2007, 595,128.

4. Periago, J. F.; Zambudio, A.; Prado, C.; J. Chromatogr., A 1997, 778, 263.

5. Zoccolillo, L.; Alessandrelli, M.; Felli, M.; Chromatographia 2001, 54,
659

6. International Agency for Research on Cancer (IARC); Monographs on the Evaluation of Carcinogenic Risk to Humans, France, 1998, vol. 45.

7. International Agency for Research on Cancer (IARC); Monographs on the Evaluation of Carcinogenic Risk to Humans, France, 1998, vol. 46.

8. http://epa.gov, acessada em Novembro 2007.

9. http://petrobras.com.br, acessada em Novembro 2007.

10. http://anp.gov.br, acessada em Novembro 2007.

11. Martines, C.; Longo, M.; Lerda, D.; Ceroni, G.; Cavallaro, A.; J. Chromatogr. Sci. 1996, 34, 413.

12. Moreira, L. S.; d’Avila, L.A.; Azevedo, D. A.; Chromatographia 2003 , $58,501$.

13. Pavlova, A.; Ivanova. R.; Acta Chomatographica 2003, 13, 215.

14. Wang, H.; Xu, H.; Guan, Y.; J. Chromatogr., A 2002, 972, 253.

15. Diehl, J. W.; di Sanzo, F. P.; J. Chromatogr., A 2005, 1080, 157.

16. Frysinger, G. S.; Gaines, R. B.; Ledford, E. B., Jr.; J. High Resol. Chromatogr. 1999, 22, 195.

17. American Society for Testing and Materials; Annual Book of ASTM Standards, ASTM International: Philadelphia, 2004, vol. 05.02, D3606.

18 American Society for Testing and Materials; Annual Book of ASTM Standards, ASTM International: Philadelphia, 1999, vol. 05.02, D4420.

19. Ref. 17, D5443.

20. Ref. 17, D5580.

21. Ref. 17, D5769.

22. Skoog, D. A.; Holler, F. J.; Nieman, T. A.; Princípios de Análise Instrumental, $5^{\text {a }}$ ed., Bookman: Porto Alegre, 2002.

23. Skoog, D. A.; West, D. M.; Holler, F.J.; Crouch, S. R.; Fundamentos de Química Analítica, $8^{\mathrm{a}}$ ed., Thomson: São Paulo, 2006.

24. Ciola, R.; Fundamentos da Cromatografia a Gás, 2ª ed., Edgard Blucher: São Paulo, 1985.

25. Lanças, F. M.; Validação de Métodos Cromatográficos de Análise, 1 ed., RIMA: São Carlos, 2004.

26. Ribani, M.; Bottoli, C. B. G.; Collins, C.H.; Jardim, I. C. S. F.; Melo, L.F.C.; Quim. Nova 2004, 27, 771.

27. Collins, C. H.; Braga, G. L.; Introdução a Métodos Cromatográficos, $2^{\mathrm{a}}$ ed., Unicamp: Campinas, 2006.

28. Lanças, F. M.; Cromatografia em Fase Gasosa, $1^{\text {a }}$ ed., Acta: São Carlos, 1993.

29. Moita Neto, J. M; Moita. G. C.; Quim. Nova 1998, 21, 467.

30. Flumignan, D. L.; Dissertação de Mestrado, Universidade Estadual Paulista Júlio de Mesquita Filho, Brasil, 2005.

31. Flumignan, D. L.; Ferreira, F. O.; Tininis, A. G.; Lopes, M. N.; de Oliveira, J. E.; J. Chromatogr., A 2008, 1202, 181.

32. Instituto Nacional de Metrologia, Normalização e Qualidade Industrial (INMETRO); Orientações sobre Validação de Métodos de Ensaios Químicos, DOQ-CGCRE-008, 2007.

33. Cooperation International Traceability in Analytical Chemistry (ITAC) and Analytical Chemistry in Europe (EURACHEM); Guide To Quality In Analytical Chemistry an Aid to Accreditation, CITAC an Eurochem, 2002. 


\section{DETERMINAÇÃO DE BENZENO, TOLUENO, ETILBENZENO E XILENOS EM GASOLINA COMERCIALIZADA NOS POSTOS DO ESTADO DO PIAUÍ}

Flamys Lena do N. Silva, José Ribeiro dos Santos Jr., José Machado Moita Neto e Rosa Lina G. do N. P. da Silva*

Departamento de Química, Universidade Federal do Piauí, 64049-550 Teresina - PI, Brasil

Danilo Luiz Flumignan e José Eduardo de Oliveira

Centro de Monitoramento e Pesquisa da Qualidade de Combustíveis, Biocombustíveis, Petróleo e Derivados, Departamento de Química Orgânica, Instituto de Química, Universidade Estadual Paulista “Júlio de Mesquita Filho”, 14800-900 Araraquara - SP, Brasil

Tabela 1S. Normas padrões ASTM para análise de gasolina

\begin{tabular}{|c|c|}
\hline Método ASTM & Título \\
\hline D 3606 & Determination of Benzene and Toluene in Finished Motor and Aviation Gasoline by Gas Chromatography \\
\hline D 4420 & Determination of Aromatics in Finished Gasoline by Gas Chromatography \\
\hline D 5443 & $\begin{array}{l}\text { Paraffin, Naphthene, and Aromatic Hydrocarbon Type Analysis in Petroleum Distillates Through } 200^{\circ} \mathrm{C} \text { by } \\
\text { Multi-Dimensional Gas Chromatography }\end{array}$ \\
\hline D 5580 & $\begin{array}{c}\text { Determination of Benzene, Toluene, Ethylbenzene, p/m-Xylene, o-Xylene, } C_{9} \text { and Heavier Aromatics, and Total Aromatics } \\
\text { in Finished Gasoline by Gas Chromatography }\end{array}$ \\
\hline D 5769 & $\begin{array}{c}\text { Determination of Benzene, Toluene, and Total Aromatics in Finished Gasolines by Gas Chromatography/ } \\
\text { Mass Spectrometry }\end{array}$ \\
\hline
\end{tabular}

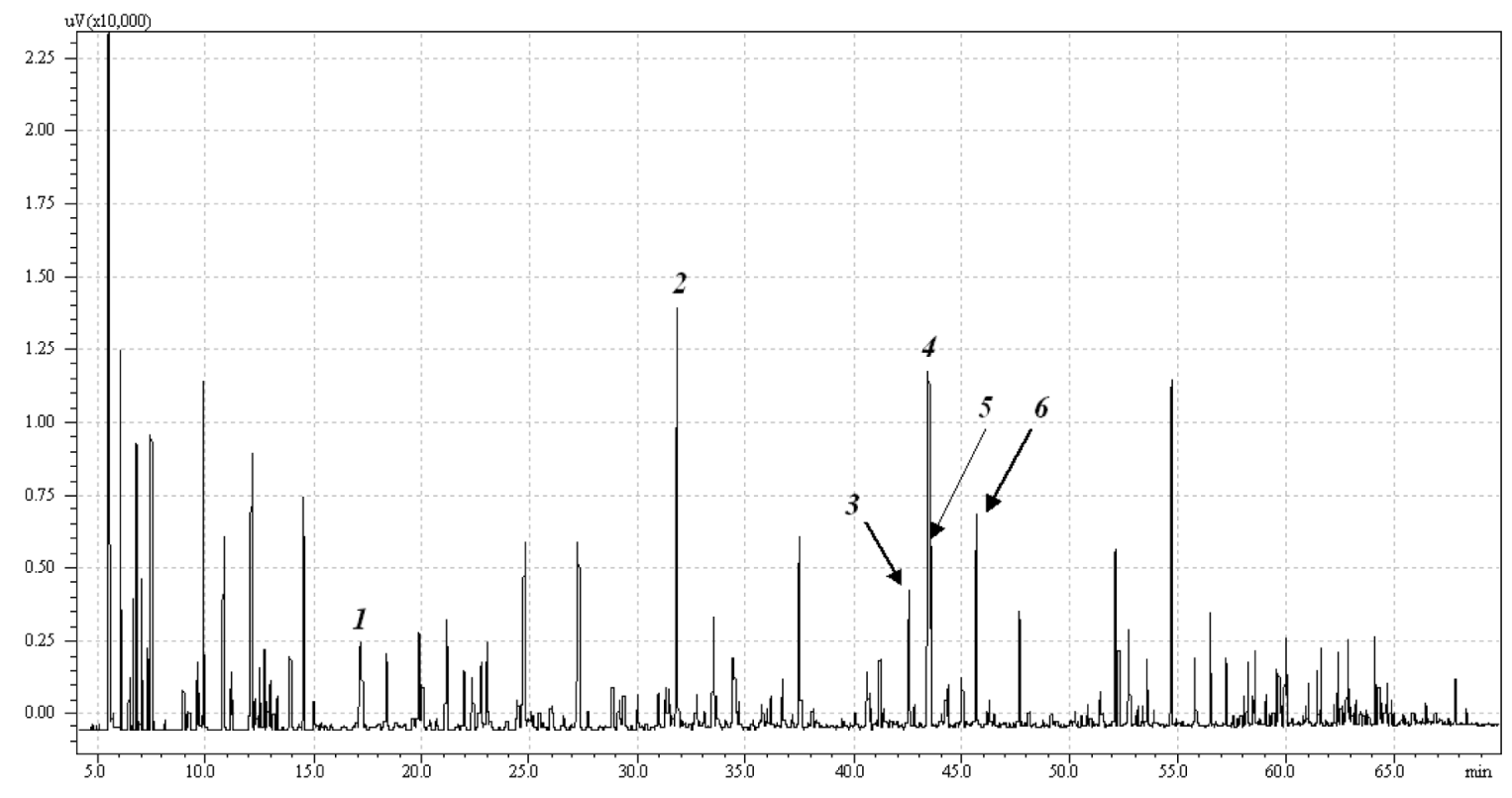

(1) benzeno $-t_{r}: 17,2 \mathrm{~min}$; (2) tolueno $-t_{r}: 31,6 \mathrm{~min}$; (3) etilbenzeno $-t_{r}: 42,5 \mathrm{~min}$; e xilenos: (4) m-xileno $-t_{r}: 43,4 \mathrm{~min}$; (5) p-xileno $-t_{r}: 43,5$ min; $e(6)$ o-xileno $-t_{r}: 45,6$ min

Figura 1S. Cromatograma típico de uma amostra de gasolina comercial brasileira com os respectivos tempos de retenção dos compostos aromáticos estudados 